\title{
Characteristics of Coal Tar Pitch-Based Mesophase Separated by High-Temperature Centrifugal Method
}

\author{
C.J. Kim, S.J. In, Y.G. Jo, S.K. Ryu and B.S. Rhee \\ (Received February 8, 1992) \\ Department of Chemical Engineering, Chungnam National University, \\ Taejon 305-764, Korea
}

\begin{abstract}
Mesophase pitches which have different amount of mesophase were prepared and separated into mesophase and isotropic phases by a high-temperature centrifugal method. Properties of mesophase during formation, growth, and coalescence of mesophase spheres were investigated by measuring the solubility, density, $\mathrm{X}$-ray diffraction, and thermogravimetric analysis.

QI was the main component of smaller mesophase spheres, and the $\beta$-resin of the isotropic matrix contributed to the growth of spheres. The density and $\mathrm{C} / \mathrm{H}$ atomic ratio of mesophase were nearly constant at $1.385 \mathrm{~g} / \mathrm{cm}^{3}$ and 2.00 , respectively, during heat-treatment. The amount of functional groups of bigger spheres was larger than smaller spheres or bulk phase. Bigger spheres have better crystallite structure than smaller spheres or bulk phase.
\end{abstract}

KEYWORDS: Mesophase sphere, High-temperature centrifugal method, Bulk phase

\section{Introduction}

A large number of studies on the mesophase formation during the heat treatment of pitches have been undertaken to investigate the properties of mesophase ${ }^{1-3)}$.

Usually the solvent extraction method ${ }^{2)}$ has been used to separate mesophase from a multi-phase mixture. However, the solvent extraction method is not adequate to get accurate information about mesophase, because the amount of mesophase in pitch is not equal to the solvent insolubles of the pitch $^{4)}$. Several researchers ${ }^{3), 5)}$ have used a high-temperature centrifugal method for the separation of mesophase from multi-phase mixtures, and for the characterization of both the mesophase and the isotropic phase. But the property changes of mesophase during formation, growth, and coalescence of mesophase spheres are not well understood as yet.

In this study, the physical and chemical properties of mesophase were investigated during formation, growth, and coalescence of mesophase spheres. The high-temperature centrifugal method was used to separate mesophase spheres from multi-phase mixtures.

\section{Experimental}

\subsection{Preparation of Mesophase Pitches}

QI-free coal tar pitch, prepared by THF extraction of raw material, was used as a precursor material for preparation of mesophase pitches. The properties of raw and QI-free coal tar pitches are shown in Table 1.

Table 1 Properties of precursor pitches

\begin{tabular}{|c|c|c|c|c|c|c|}
\hline \multirow{2}{*}{\begin{tabular}{|c|} 
Properties \\
Pitches
\end{tabular}} & \multicolumn{3}{|c|}{ Solubility (wt\%) } & \multirow{2}{*}{$\begin{array}{c}\mathrm{C} / \mathrm{H} \\
\text { atomic } \\
\text { ratio }\end{array}$} & \multirow{2}{*}{$\begin{array}{l}\text { S.P. } \\
\left({ }^{\circ} \mathrm{C}\right)\end{array}$} & \multirow{2}{*}{$\begin{array}{c}\text { Mesophase } \\
\text { content } \\
(\mathrm{vol} \%)\end{array}$} \\
\hline & HI & BI & QI & & & \\
\hline Raw & 56.68 & 27.47 & 5.46 & 1.80 & 115 & - \\
\hline THF extr & 62.33 & 16.41 & - & 1.68 & 97.5 & - \\
\hline
\end{tabular}

HI: n-Hexane Insoluble QI : Quinoline Insoluble

BI: Benzene Insoluble S.P. : Softening Point 
Mesophase pitches were prepared by the two-stage heat treatment ${ }^{6)}$ of the pitch. In the first stage of heat treatment, QI-free coal tar pitch was heated to $430^{\circ} \mathrm{C}$ at the heating rate of $5^{\circ} \mathrm{C} / \mathrm{min}$ and maintained for 2 hours at this temperature. Nitrogen was used to keep the pressure of the reactor at $10 \mathrm{kgf} / \mathrm{cm}^{2}$. During the second stage of the process, the pitch was heated to $450^{\circ} \mathrm{C}$ with nitrogen sparging, and the temperature was maintained during mesophase formation process.

\subsection{Centrifugal Separation of Mesophase Pitch}

Mesophase pitches were transferred to a hightemperature centrifuge and centrifuged at $330^{\circ} \mathrm{C}$, rotational acceleration of $250 \mathrm{~g}\left(\mathrm{~g}=9.8 \mathrm{~m} / \mathrm{sec}^{2}\right)$ for $30 \mathrm{~min}$, and then subsequently cooled. Fig. 1 shows the schematic drawing of the high-temperature centrifuge which was made by the modification of Singer's apparatus $^{3)}$.

Four centrifuge cups were screw-capped to the bars of the top of the shaft. The shaft was rotated by a variable-speed DC-motor installed just under the bottom insulation base. The inside diameter and height of the furnace were $30 \mathrm{~cm}$ and $19 \mathrm{~cm}$, respectively.

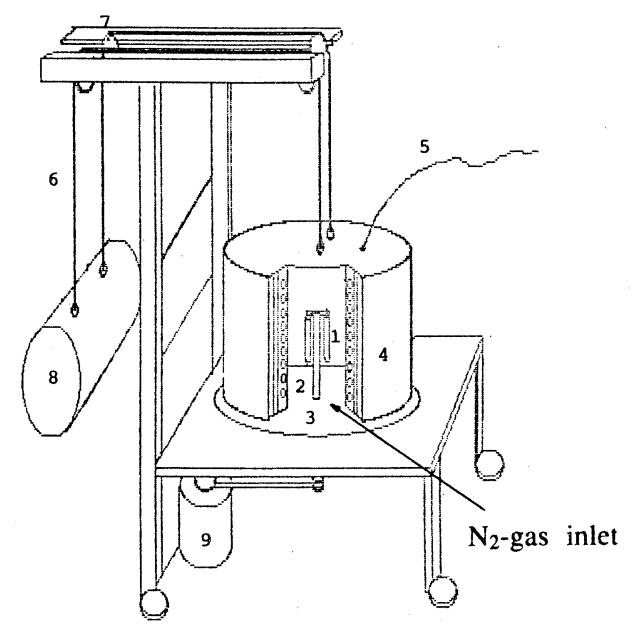
1. Sample cup
6. Counter-weight cable
2. Shaft
7. Pulley assembly
3. Insulated base
8. Counter-weight
4. Furnace
9. Motor
5. Thermocouple

Fig. 1 Schematic drawing of high-temperature centrifuge with cut way view of sample chamber.

\subsection{Analyses}

The amount of mesophase was measured by microscopy ${ }^{7)}$ and the centrifugal technique ${ }^{3)}$. The solubilities of the pitches were measured in benzene and quinoline by refluxing the solvents. Chemical composition of pitches were measured with an elemental analysis apparatus (YANACO CHN coder MT-3). Thermogravimetric analyses (TGA V1.1F, Dupont 9900 ) of the mesophases were carried out to $600^{\circ} \mathrm{C}$ (heating rate $=6^{\circ} \mathrm{C} / \mathrm{min}$ ) and $900^{\circ} \mathrm{C}$ (heating rate $=$ $10^{\circ} \mathrm{C} / \mathrm{min}$ ) in oxygen and nitrogen atmosphere, respectively. X-ray diffraction analyses were conducted for investigation of crystal structure of mesophase, and only $\mathrm{CuK} \alpha$ radiation was used. The samples were finely crushed to pass the 200 Tyler mesh. The crystallite stack height, $\mathrm{L}_{c}$, was obtained by using the Scherrer formula ${ }^{8)}$. Although the equation may not be truly applicable for pitches, the value of Lc may still provide an estimate of the number of pitch molecules with locally correlated basal orientation ${ }^{9)}$. The densities of pitches were determined using the sink-float method ${ }^{10)}$. A series of mixtures of p-xylene and carbon tetrachloride were prepared for the density measurement. The temperature of mixtures was controlled in the range of $20 \pm 0.5^{\circ} \mathrm{C}$, and the densities of mixtures were varied from $1.200 \mathrm{~g} / \mathrm{cm}^{3}$ to $1.400 \mathrm{~g} / \mathrm{cm}^{3}$, in steps of $0.005 \mathrm{~g} / \mathrm{cm}^{3}$.

\section{Results and Discussion}

\subsection{Mesophase Pitches}

Fig. 2 shows the polarized micrograph photos of mesophase pitches prepared with varying the heat soaking time in the second stage of heat treatment. It shows a typical change of formation, growth, and coalescence of mesophase spheres. When the mesophase content in pitch is over $57 \%$ (Fig. 2(c)), the shape of mesophase spheres is deformed, and matrix change between isotropic and anisotropic phases is shown between $57 \%$ (Fig. 2(c)) and $82 \%$ (Fig. 2(d)) of mesophase content. In Fig. 2(e), isotropic phase exist as small spheres in mesophase matrix.

\subsection{Centrifugal Separation of Mesophase Pitches}

Fig. 3 shows a polished longitudinal cross-section of a centrifuged sample. We can clearly recognize the interface of two phases. Though mesophase spheres below $10 \mu \mathrm{m}$ in diameter are shown in isotropic phase, 

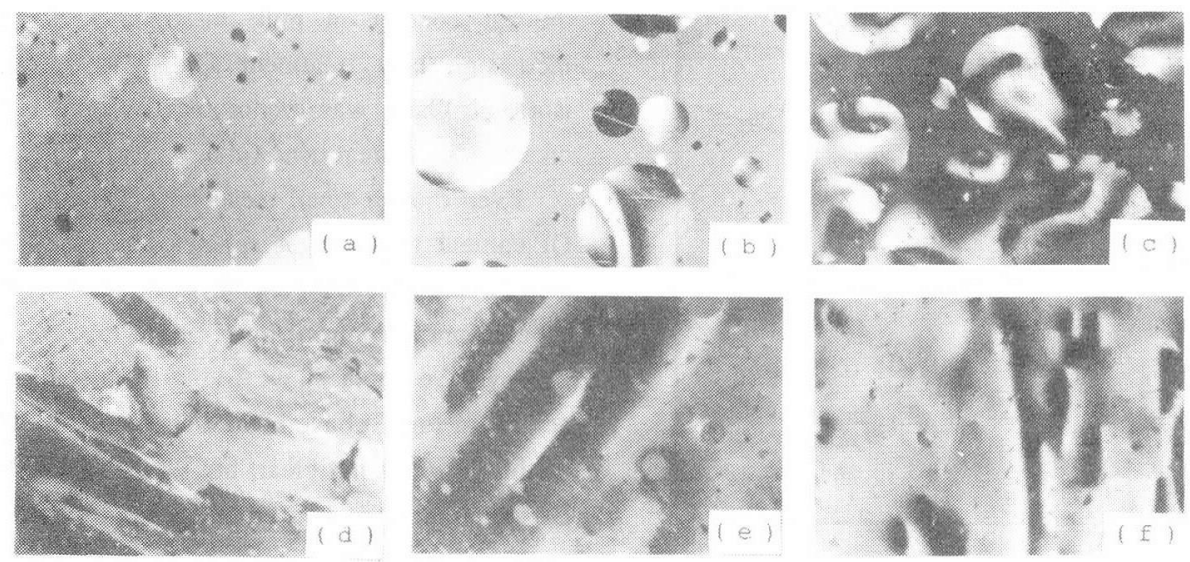

Fig. 2 Polarized micrographs of mesophase pitches: (a) $15 \mathrm{vol} \%$, (b) $34 \mathrm{vol} \%$, (c) 57 vol\%, (d) $82 \mathrm{vol} \%$, (e) $97.5 \mathrm{vol} \%$, and (f) 100 vol\% of mesophase content.

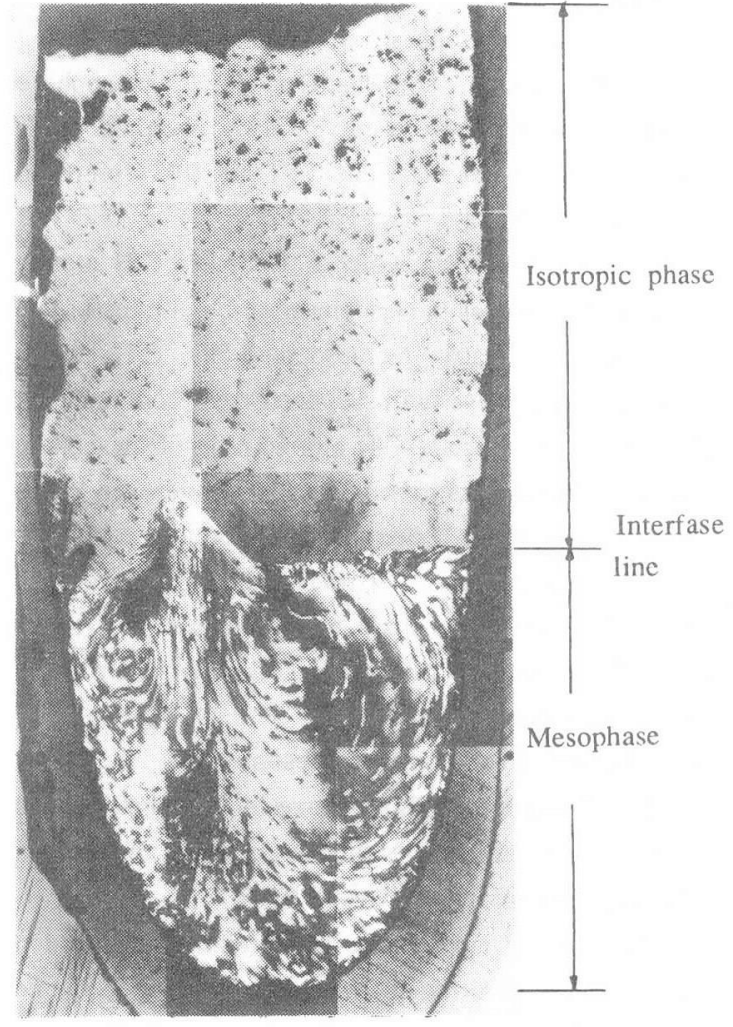

$500 \mu \mathrm{m}$

Fig. 3 Polarized micrograph of longitudinal crosssection of centrifuged mesophase pitch.

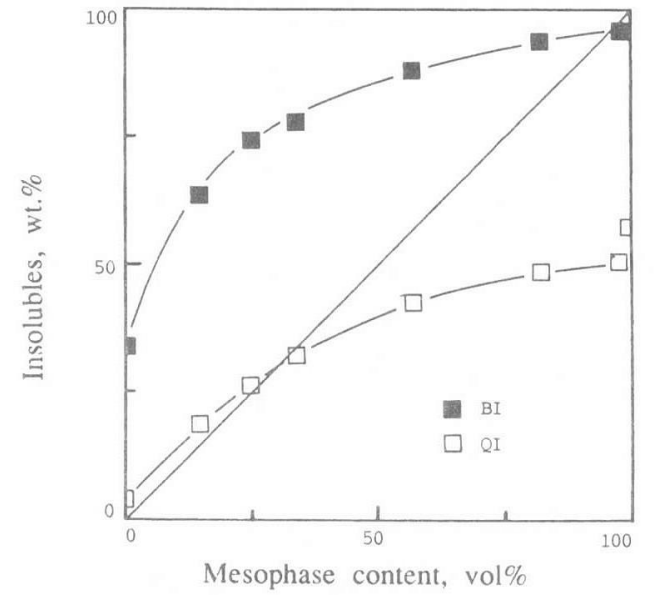

Fig. 4 Relationship between mesophase content and benzene and quinoline insolubles.

the phase separation is almost perfect. Mesophase shows orientation follow the tube wall and a vortex. The short separation time will prevent the reaction in mesophase.

\subsection{Solubility of the Pitches}

If the content of solvent insolubles in mesophase pitches are equal to the amount of mesophase, we can most likely use the solvent extraction to investigate the properties of mesophase. But as shown in Fig. 4, BI and QI contents of mesophase pitches are not consistent with the amount of mesophase. Therefore, we have tried the centrifugal separation method to get more exact 


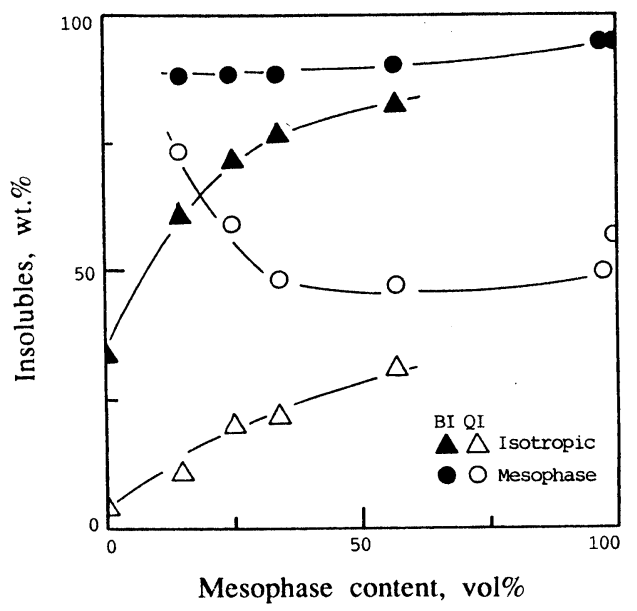

Fig. 5 Benzene and quinoline insoluble contents in separated mesophase and isotropic phase.

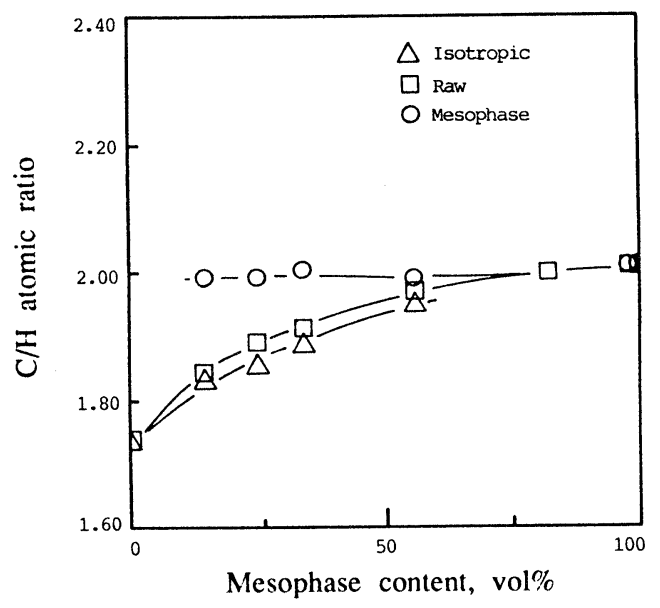

Fig. $6 \mathrm{C} / \mathrm{H}$ atomic ratio of separated mesophase and isotropic phase.

information about mesophase.

After the centrifugal separation of mesophase pitches, both benzene and quinoline insolubles in each phases were measured. As we can see in Fig. 5, small mesophase spheres were mainly consisted of quinoline insolubles. They were grown into bigger spheres by the uptake of $\beta$-resin (benzene insolubles and quinoline solubles) from the isotropic matrix as increasing the heat treatment time. When the content of mesophase in pitch was over $34 \mathrm{vol} \%$, the amount of QI in mesophase remained nearly constant at about $48 \mathrm{wt} \%$ until all the pitch was transformed to bulk phase. Also the QI content in bulk mesophase increased with increasing heat treatment time. The QI content in isotropic phase was monotonically increased with increasing heat treatment time.

Even though small mesophase spheres have higher QI content than bigger spheres and bulk phase, they also have higher BS content. In the case of mesophase spheres prepared from multi-phase mixture (a) in Fig. 2 , they have about 88 wt.\% of BI. But bulk phases, (e) and (f) in Fig. 2, have about $95 \mathrm{wt} . \%$ of BI. The reason of this result is not clear, but we can consider that the BS component is also an important construction molecule of mesophase spheres in the heat soaking system, even though BS component in the isotropic matrix will decrease the growth rate of mesophase spheres during heat treatment.

\subsection{Elemental Analyses}

Fig. 6 shows the changing of $\mathrm{C} / \mathrm{H}$ atomic ratio of separated mesophase and isotropic phase, respectively. The value for mesophase is constant at 2.00 during formation, growth, and coalescence of mesophase spheres. But the $\mathrm{C} / \mathrm{H}$ atomic ratio of isotropic phase continuously increased from 1.71 to 1.93 (prepared from (d) in Fig. 2).

If some molecules which have lower $\mathrm{C} / \mathrm{H}$ atomic ratio than molecules in mesophase were not transformed from isotropic matrix to mesophase, the $\mathrm{C} / \mathrm{H}$ atomic ratio of mesophase will be increased continuously. Therefore, the constant $\mathrm{C} / \mathrm{H}$ atomic ratio of mesophase indicates that the rate of elimination of hydrogen atoms from mesophase by a thermal condensation reaction is almost equal to the rate of transformation of hydrogen atoms from isotropic phase to mesophase. This transformation of hydrogen atoms was mainly performed by the $\beta$-resin component.

\subsection{Thermal Properties of Mesophases}

Fig. 7 and Fig. 8 show the residual weight change of mesophase with increasing temperature in nitrogen and oxygen atmosphere, respectively.

The starting temperature of thermal decomposition of mesophase in nitrogen atmosphere is being increased with transforming from small spheres to bulk phase. However, in case of weight change during heating to $600^{\circ} \mathrm{C}$, mesophases which were prepared from (a), (e), and (f) in Fig. 2 show higher residual weight than mesophases prepared from (b) and (c) in Fig. 2. 


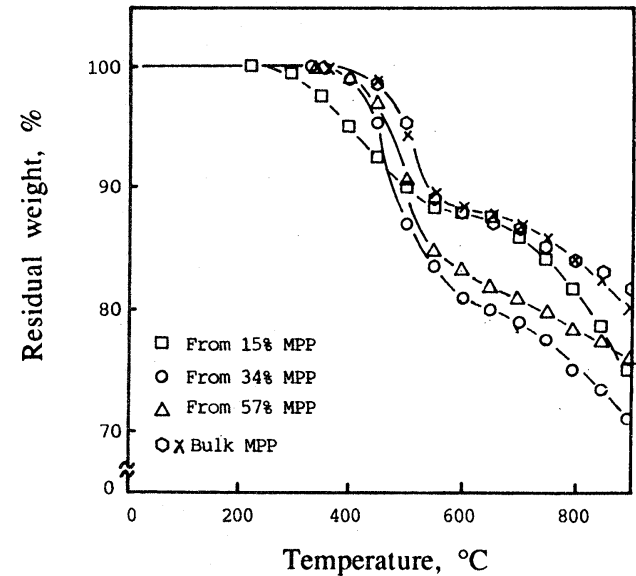

Fig. 7 TGA of separated mesophases in nitrogen atmosphere.

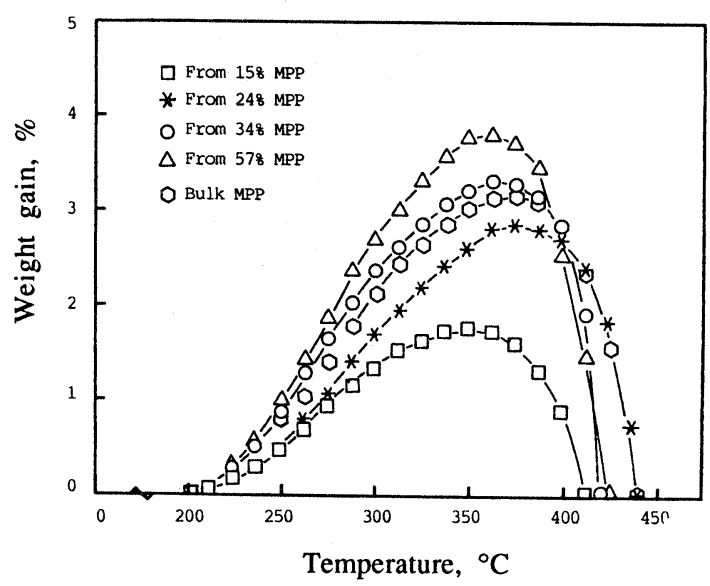

Fig. 8 TGA of separated mesophases in oxygen atmosphere.

In case of weight change during heating in oxygen atmosphere, mesophases prepared from (b) and (c) in Fig. 2 show higher weight gain than mesophases prepared from (a), (e), and (f) in Fig. 2.

This implies that bigger mesophase spheres have more plentiful functional groups than smaller spheres or bulk phase, but in the case of smaller spheres, smaller molecules present which will most likely exist as benzene soluble components. In fact, from solubility measurement we can conclude that the BS content of smaller spheres is higher than that of bigger spheres or bulk phase.

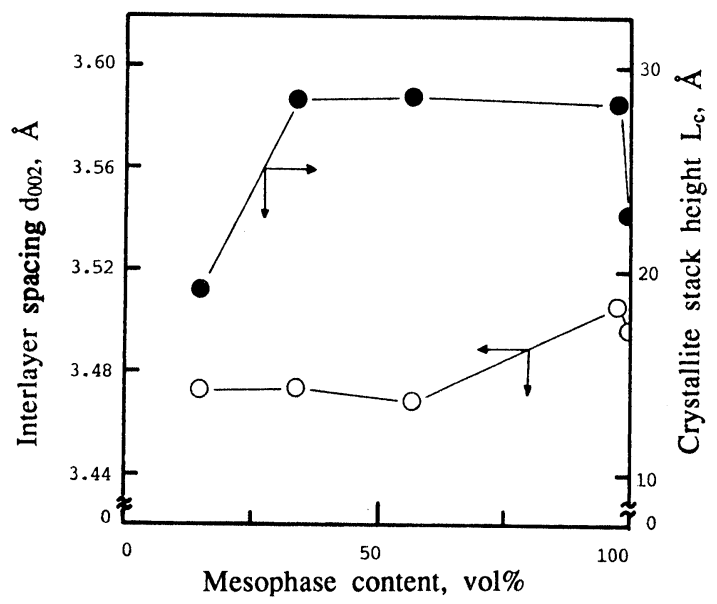

Fig. 9 Interlayer spacing and crystallite stack height of separated mesophases.

\subsection{Crystal Structure in Mesophase}

Fig. 9 shows the change of crystallite structure in mesophase during formation, growth, and coalescence of mesophase spheres. The interlayer spacing of spheres is almost constant at $3.47 \AA$. As the shape of mesophase domains change from sphere to bulk phase, the interlayer spacing of mesophase is increased from 3.47 $\AA$ to $3.50 \AA$. However, the crystallite stack height $\left(\mathrm{L}_{\mathrm{c}}\right)$ of spheres is increased from $19 \AA$ to $28 \AA$, when changing from smaller sphere to bigger ones. However, for bulk phase, the value is decreased to $22 \AA$ because of the second thermal decomposition ${ }^{11)}$.

The reason of these complex trends is not clear, but it is probably due to the transformation of some molecules from isotropic matrix to mesophase and to the coalescence among mesophase spheres which will increase the structural defects of mesophase.

\subsection{Density Changes}

Fig. 10 shows the density difference between mesophase and isotropic phase. During formation, growth, coalescence, and changing to bulk phase of mesophase spheres, the density is kept at about $1.385 \mathrm{~g} / \mathrm{cm}^{3}$. But the density of isotropic phase is increased from $1.300 \mathrm{~g} / \mathrm{cm}^{3}$ to $1.370 \mathrm{~g} / \mathrm{cm}^{3}$ (prepared from (d) in Fig. 2) with increasing heat treatment time, and it is due to the increasing of molecular size. These results are very similar with that of Machnikowski ${ }^{12)}$, though he have studied on the solvent extraction of petroleum pitch. 


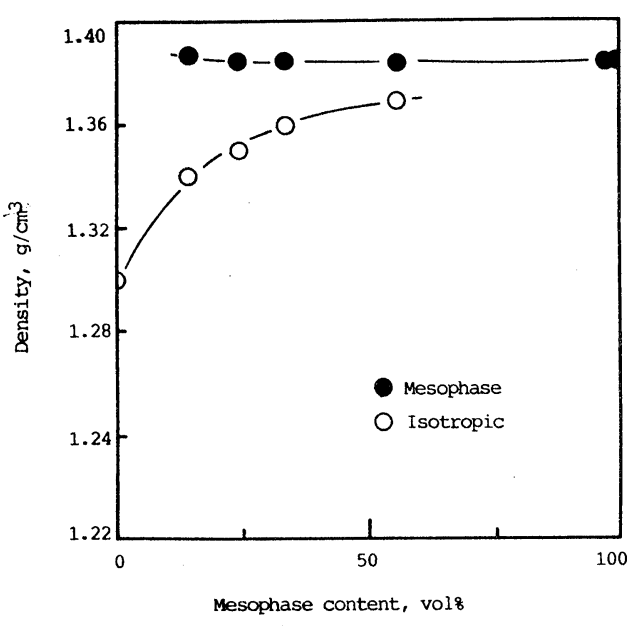

Fig. 10 Densities of separated mesophase and isotropic phase.

\section{Conclusion}

Coal tar pitch-based mesophase pitches were prepared and separated into mesophase and isotropic phase by the high-temperature centrifugal method. Properties of mesophase during formation, growth, and coalescence of spheres were investigated by several analyses, and the following results were obtained.

QI was the main component of smaller mesophase spheres, and $\beta$-resin of isotropic matrix contributed to the growth of spheres. The $\mathrm{C} / \mathrm{H}$ atomic ratio of mesophase was almost constant at 2.00 during change from smaller spheres to bulk phase. This indicates that the rate of elimination of hydrogen atoms from mesophase by a thermal condensation reaction is almost equal to the rate of transformation of hydrogen atoms from isotropic phase to mesophase. However, the $\mathrm{C} / \mathrm{H}$ atomic ratio of isotropic matrix was increased from 1.71 to 1.93 (prepared from (d) in Fig. 2). Bigger mesophase spheres have more functional groups than smaller spheres or bulk phase, but in the case of these smaller spheres, smaller molecules present which will most likely exist as BS components. The interlayer spacing of mesophase spheres is almost constant at $3.47 \AA$. As the shape of mesophase domains change from sphere to bulk phase, the interlayer spacing of mesophase is increased from $3.47 \AA$ to $3.50 \AA$. However, the crystallite stack height $\left(\mathrm{L}_{\mathrm{c}}\right)$ of spheres is increased from $19 \AA$ to $28 \AA$, when changing from smaller sphere to bigger ones. The density of mesophase remained constant at $1.385 \mathrm{~g} / \mathrm{cm}^{3}$ during the change from smaller spheres to bulk phase. However, the density of isotropic phase increased from $1.300 \mathrm{~g} / \mathrm{cm}^{3}$ to $1.370 \mathrm{~g} / \mathrm{cm}^{3}$ (prepared from (d) in Fig. 2).

\section{References}

1) G.D. Brooks and G.H. Taylor, Carbon 3, 185 (1965).

2) H. Honda, H. Kimura and Y. Sanada, Carbon 9, 695 (1971).

3) L.S. Singer, D.M. Riffle and A.R. Cherry, Carbon 25, 249 (1987).

4) S. Chwastiak and J.L. Lewis, Carbon 16, 156 (1978).

5) S.H. Chen and R.J. Diefendorf, International Carbon Conference (1984).

6) B.S. Rhee, D.H. Chung, S.J. In and D.D. Edie, Carbon 29, 343 (1991).

7) S. Chwastiak, R.T. Lewis and J.D. Ruggiero, Carbon 19, 357 (1981).

8) J.B. Donnet and R.C. Bansal, "Carbon Fibers" Marcel Dekker, Inc. (1984) pp. 58.

9) W.C. Stevens, S.H. Chen and R.J. Diefendorf, International Carbon Conference (1987).

10) K.J. Hüttinger and J.P. Wang, Carbon 29, 439 (1991).

11) Y. Hishiyama and Y. Kaburagi, International Carbon Conference (1984).

12) J. Machnikowski and L. Polec, International Carbon Conference (1984). 